2. - Types of ultrafilters, Proc. of the 2nd Topology Symposium 1966, Academia 1967, Praha.

3. M. Katětov, A theorem on mappings, Comment Math. Univ. Carolinae. 8 (1967), 431-433.

4. R. Raimi, Homeomorphisms and invariant measures for $\beta N-N$, Duke Math. J. 13 (1966), 1-12.

5. W. Rudin, Homogeneity problems in the theory of Cech compactification, Duke Math. J. 23 (1956), 409-420.

Louisiana State University, Baton Rouge, University of California, Riverside, and Mathematics Institute, Praha

\title{
REPRESENTATION OF FRACTIONAL POWERS OF INFINITESIMAL GENERATORS OF SEMIGROUPS
}

BY H. BERENS, P. L. BUTZER AND U. WESTPHAL ${ }^{1}$

Communicated by Felix Browder, September 1, 1967

1. Introduction. Let $X$ be a real or complex Banach space and $\mathbb{E}(X)$ the Banach algebra of endomorphisms of $X$. Let $\{T(u) ; u \geqq 0\}$ be an equibounded semigroup of operators of class $\left(\mathfrak{G}_{0}\right)$ in $\mathbb{E}(X)$ with infinitesimal generator $A . A$ is a closed linear operator with domain $D(A)$ dense in $X$. For these concepts see e.g. E. Hille-R. S. Phillips $[5$, Chapters X-XII].

One purpose of this note is to give a representation for the fractional power $(-A)^{\gamma}, \gamma>0$, of the operator $(-A)$. The result obtained will be a generalization of one due to J. L. Lions-J. Peetre [7, Chapter VII,\$2]: Let $\gamma$ be a positive integer. An element $f \in X$ belongs to $D\left((-A)^{\gamma}\right)$ if and only if the integral

$$
\frac{1}{C_{\gamma, r}} \int_{\epsilon}^{\infty} \frac{[I-T(u)]^{r f}}{u^{1+\gamma}} d u \quad(\epsilon>0 ; r \text { any integer }>\gamma) .
$$

converges in norm as $\epsilon \rightarrow 0_{+}$, the constant $C_{\gamma, r}$ being given by

$$
C_{\gamma, r}=\int_{0}^{\infty} \frac{\left(1-e^{-u}\right)^{r}}{u^{1+\gamma}} d u .
$$

The limit is then equal to $(-A)^{\gamma} f$.

1 The research of this author was supported by a DFG grant. 
We show that this result holds for arbitrary $\gamma>0$. The proof indicated below has its origin in a paper of $U$. Westphal [8] on fractional derivatives of functions in $L^{p}(0, \infty), 1 \leqq p<\infty$, in connection with the semigroup of right translations; the author uses Laplace transform methods (see also H. Berens-U. Westphal [3]).

Of the various methods in defining the fractional power $(-A)^{r}$, $\gamma>0$, of a closed linear operator $A$ (cf. A. V. Balakrishnan [1], [2], H. Komatsu [6], K. Yosida [9, Chapter IX,\$11]) we follow the one by Balakrishnan [2] but restrict ourselves ab initio to the case that $A$ is the generator of the semigroup $\{T(u) ; u \geqq 0\}$ under consideration. Under these assumptions the resolvent $R(\lambda ; A)$ of $A$ exists for each $\lambda>0$ and $\|R(\lambda ; A) f\| \leqq M\|f\|$ for all $f \in X$ uniformly with respect to $\lambda>0, M$ being some positive constant. For $\gamma>0$ with $k-1<\gamma<k$, $k=1,2, \cdots$, we define the linear operator $J^{\gamma}$ on $D\left(A^{k}\right)$ by

$$
J^{\gamma} f=-\frac{\sin \gamma \pi}{\pi} \int_{0}^{\infty} \lambda^{\gamma-k} R(\lambda ; A) A^{k} f d \lambda .
$$

$J^{\gamma}$ is preclosed and has the usual properties of fractional powers. The operator $(-A)^{\gamma}$ is then defined to be the smallest closed extension of $J^{\gamma}$. It is easily verified that for each $f \in D\left(A^{k}\right)$ and $k-1 \leqq \gamma<k$ $(\gamma=0$ excluded $)(-A)^{\gamma} f$ has the representation

$$
(-A)^{\gamma} f=\frac{1}{C_{\gamma, k}} \int_{0}^{\infty} \frac{[I-T(u)]^{k} f}{u^{1+\gamma}} d u,
$$

where $C_{\gamma, k}$ is given by (2).

We only present here our main results. The full details will be published elsewhere.

2. Fundamental lemma. The main new tool is

Lemma. Let $0<\gamma<r, r=1,2, \cdots$. For all $f \in X$ the integral

$$
\int_{0}^{\infty} T(u) f q_{\gamma, r}(u) d u
$$

belongs to $D\left((-A)^{\gamma}\right)$ and

$$
(-A)^{\gamma}\left[\int_{0}^{\infty} T(u) f q_{\gamma, r}\left(\frac{u}{\epsilon}\right) \frac{d u}{\epsilon}\right]=\int_{\epsilon}^{\infty} \frac{[I-T(u)]^{r} f}{u^{1+\gamma}} d u \quad(\epsilon>0),
$$

where $q_{\gamma, r}(u)$ is defined by its Laplace transform

$$
\int_{0}^{\infty} e^{-s u} q_{\gamma, r}(u) d u=\frac{1}{s^{\gamma}} \int_{1}^{\infty} \frac{\left(1-e^{-s u}\right)^{r}}{u^{1+\gamma}} d u \quad(\operatorname{Re} s>0) .
$$


Sketch of Proof. Introducing the auxiliary functions

$$
a(u)=\frac{u^{\gamma-1}}{\Gamma(\gamma)} \quad(u>0), \quad b_{\epsilon}(u)=\left\{\begin{array}{l}
0,0<u \leqq \epsilon, \\
u^{-1-\gamma}, u>\epsilon,
\end{array} \quad(\epsilon>0),\right.
$$

the function $q_{\gamma, r}(u)$ has the representation

$$
q_{\gamma, r}(u)=\sum_{i=1}^{r}(-1)^{i}\left(\begin{array}{c}
r \\
i
\end{array}\right)\left\{i^{\gamma}\left[a * b_{i}\right](u)-\frac{1}{\gamma} a(u)\right\},
$$

where $\left[a * b_{i}\right](u)$ denotes the Laplace convolution of $a(u)$ and $b_{i}(u)$. Note that $q_{\gamma, r}(u)$ belongs to $L^{1}(0, \infty)$ with $\int_{0}^{\infty} q_{\gamma, r}(u) d u=C_{\gamma, r}$. Thus the integral (4) exists in the sense of Bochner and

$$
\mathrm{s}-\lim _{\epsilon \rightarrow 0^{+}} \int_{0}^{\infty} T(u) f q_{\gamma, r}\left(\frac{u}{\epsilon}\right) \frac{d u}{\epsilon}=C_{\gamma, r} f \quad(f \in X) .
$$

For any fixed real $\gamma>0$, choose the integer $k$ such that $k-1 \leqq \gamma<k$ and let the integer $r \geqq k$. Then for each $f \in X$ and $\epsilon, \eta>0$ the following fundamental identity holds:

$$
\begin{aligned}
\int_{\eta}^{\infty} \frac{[I-T(v)]^{k}}{v^{1+\gamma}} d v & \int_{0}^{\infty} T(u) f q_{\gamma, r}\left(\frac{u}{\epsilon}\right) \frac{d u}{\epsilon} \\
& =\int_{0}^{\infty} \frac{[I-T(v)]^{r}}{v^{1+\gamma}} d v \int_{0}^{\infty} T(u) f q_{\gamma, k}\left(\frac{u}{\eta}\right) \frac{d u}{\eta} .
\end{aligned}
$$

By (7) the right-hand side of (8) is strongly convergent as $\eta \rightarrow 0_{+}$and

$$
\begin{aligned}
\mathrm{s}-\lim _{\eta \rightarrow 0^{+}} \frac{1}{C_{\gamma, k}} \int_{\eta}^{\infty} \frac{[I-T(v)]^{k}}{v^{1+\gamma}} d v \int_{0}^{\infty} T(u) f q_{\gamma, r} & \left(\frac{u}{\epsilon}\right) \frac{d u}{\epsilon} \\
& =\int_{\epsilon}^{\infty} \frac{[I-T(v)]^{r} f}{v^{1+\gamma}} d v .
\end{aligned}
$$

Then in case $f \in D\left(A^{k}\right)$, relation (3) gives the result (5) for all $f \in D\left(A^{k}\right)$. Since $D\left(A^{k}\right)$ is dense in $X$ and $(-A)^{\gamma}$ is a closed operator, (5) finally holds for all $f \in X$.

It remains to prove the identity (8). Using the representation (6) of $q_{\gamma, k}(u)$, the right-hand side of (8) can be rewritten in the form

$$
\begin{aligned}
& \sum_{j=1}^{r} \sum_{i=1}^{k}(-1)^{j+i}\left(\begin{array}{l}
r \\
j
\end{array}\right)\left(\begin{array}{l}
k \\
i
\end{array}\right) j^{\gamma} \int_{0}^{\infty}[T(v)-I] b_{j \mathrm{\epsilon}}(v) d v \\
& \cdot \int_{0}^{\infty} T(u) f\left\{i^{\gamma}\left[a * b_{i \eta}\right](u)-\frac{1}{\gamma \eta^{\gamma}} a(u)\right\} d u .
\end{aligned}
$$

Setting 


$$
I_{1} \equiv j^{\gamma} \int_{0}^{\infty} T(v) b_{j \epsilon}(v) d v \int_{0}^{\infty} T(u) f\left\{i^{\gamma}\left[a * b_{i \eta}\right](u)-\frac{1}{\gamma \eta^{\gamma}} a(u)\right\} d u
$$

and interchanging the order of integration, then

$$
\begin{aligned}
I_{1} & =j^{\gamma} \int_{0}^{\infty} T(u) f d u \int_{0}^{u} b_{j_{e}}(v)\left\{i^{\gamma}\left[a * b_{i \eta}\right](u-v)-\frac{1}{\gamma \eta^{\gamma}} a(u-v)\right\} d v \\
& =\int_{0}^{\infty} T(u) f\left\{(i j)^{\gamma}\left[a * b_{i \eta} * b_{j e}\right](u)-\frac{j^{\gamma}}{\gamma \eta^{\gamma}}\left[a * b_{j e}\right](u)\right\} d u .
\end{aligned}
$$

Furthermore,

$$
\begin{aligned}
I_{2} & \equiv j^{\gamma} \int_{0}^{\infty} b_{j \epsilon}(v) d v \int_{0}^{\infty} T(u) f\left\{i^{\gamma}\left[a * b_{i \eta}\right](u)-\frac{1}{\gamma \eta^{\gamma}} a(u)\right\} d u \\
& =\int_{0}^{\infty} T(u) f\left\{\frac{i^{\gamma}}{\gamma \epsilon^{\gamma}}\left[a * b_{i \eta}\right](u)-\frac{1}{\gamma^{2} \epsilon^{\gamma} \eta^{\gamma}} a(u)\right\} d u .
\end{aligned}
$$

Since the difference $I_{1}-I_{2}$ is symmetric in the pairs $(\epsilon, j)$ and $(\eta, i)$, this proves that the right-hand side of (8) is equal to the left-hand one.

REMARK. The relation (5) and the identity (8) are counterparts of the relation

$$
A\left[\frac{1}{u} \int_{0}^{u} T(v) f d v\right]=\frac{T(u)-I}{u} f, \quad(u>0 ; f \in X),
$$

and the identity

$$
\begin{array}{r}
\frac{T(t)-I}{t}\left[\frac{1}{u} \int_{0}^{u} T(v) f d v\right]=\frac{1}{t} \int_{0}^{t} T(v) \frac{[T(u)-I] f}{u} d v \\
(t, u>0 ; f \in X)
\end{array}
$$

for the operator $(-A)^{\gamma}$. These two simple equations are cornerstones of semigroup theory.

3. Representation theorems. Let us now formulate some of the many possible applications of the lemma.

TheOREM 1. Let $0<\gamma<r, r=1,2, \cdots$. An element $f \in X$ belongs to the domain of $(-A)^{\gamma}$ if and only if the integral (1) converges strongly as $\epsilon \rightarrow 0^{+}$. In this case we have

$$
(-A)^{\gamma} f=\operatorname{s}_{\epsilon \rightarrow 0^{+}} \frac{1}{C_{\gamma, r}} \int_{e}^{\infty} \frac{[I-T(u)]^{r} f}{u^{1+\gamma}} d u .
$$


This theorem is the generalization of the result of Lions-Peetre [7] to arbitrary $\gamma>0$. We note that the proof given by the latter authors in their particular instance is entirely different from ours and uses distribution theory.

Corollary. If $X$ is reflexive, then the condition that the integral (1) converges strongly for $\epsilon \rightarrow 0^{+}$may be replaced by

$$
\left\|\int_{\epsilon}^{\infty} \frac{[I-T(u)]^{r} f}{u^{1+\gamma}} d u\right\|=O(1) \quad\left(\epsilon \rightarrow 0_{+}\right) .
$$

The proof follows by a result of P. L. Butzer (see $[4, \S 2.1]$ ).

Theorem 2. An $f \in X$ belongs to $D\left((-A)^{\gamma}\right), k-1<\gamma<k, k=1$, $2, \cdots$, if and only if

$$
\mathrm{s}-\lim _{\epsilon \rightarrow 0^{+}} \frac{1}{\Gamma(-\gamma)} \int_{\epsilon}^{\infty}\left\{T(u) f-\sum_{i=0}^{k-1} \frac{u^{i}}{i !} A^{i f}\right\} \frac{d u}{u^{1+\gamma}}
$$

exists, the limit then being equal to $(-A)^{\gamma} f$.

This is a generalization of a result in [1].

Let $X^{*}$ be the dual space of $X$ and $\left\{T^{*}(u) ; u \geqq 0\right\}$ the dual semigroup in $\&\left(X^{*}\right)$. Set

$$
X_{0}^{*}=\left\{f^{*} \in X^{*} ; \lim _{u \rightarrow 0^{+}}\left\|T^{*}(u) f^{*}-f^{*}\right\|=0\right\} .
$$

Concerning fractional powers of the dual operator $\left(-A^{*}\right)$ of $(-A)$ we have

Theorem 3. Let $0<\gamma<r, r=1,2, \cdots$. For an $f^{*} \in X^{*}$ the following assertions are equivalent:

(i) $f^{*} \in D\left(\left(-A^{*}\right)^{r}\right)$.

(ii) $f^{*} \in X_{0}^{*}$ and the weak $k^{*}$ limit of

$$
\frac{1}{C_{\gamma, r}} \int_{0}^{\infty} \frac{\left[I^{*}-T^{*}(u)\right]^{r} f^{*}}{u^{1+\gamma}} d u
$$

exists as $\epsilon \rightarrow 0_{+}$, the limit being equal to $\left(-A^{*}\right)^{\gamma} f^{*}$.

(iii) $f^{*} \in X_{0}^{*}$ and

$$
\left\|\frac{1}{C_{\gamma, r}} \int_{e}^{\infty} \frac{\left[I^{*}-T^{*}(u)\right] f^{*}}{u^{1+\gamma}} d u\right\|=O(1) \quad\left(\epsilon \rightarrow 0_{+}\right) .
$$

The equivalence (iii) depends upon a result due to $\mathrm{K}$. de Leeuw (see $[4, \S 2.1]$ ). 


\section{REFERENCES}

1. A. V. Balakrishnan, An operational calculus for infinitesimal generators of semigroups, Trans. Amer. Math. Soc. 91 (1959), 330-353.

2. - Fractional powers of closed operators and the semigroups generated by them, Pacific J. Math. 10 (1960), 419-437.

3. H. Berens and U. Westphal, Zur Charakterisierung von Ableitungen nichtganzer Ordnung im Rahmen der Laplacetransformation, Math. Nachr. (in press).

4. P. L. Butzer and H. Berens, Semi-groups of operators and approximation, Grundlehren d. math. Wiss., vol. 145, Springer, New York 1967.

5. E. Hille and R. S. Phillips, Functional analysis and Semi-groups, Amer. Math. Soc. Colloq. Publ. Vol. 31, rev. ed., Amer. Math. Soc., Providence, R. I., 1957.

6. H. Komatsu, Fractional powers of operators, Pacific J. Math. 19 (1966), 285-346.

7. J. L. Lions and J. Peetre, Sur une classe d'espaces d'interpolation, Inst. Hautes Etudes Sci. Publ. Math. 19 (1964), 5-68.

8. U. Westphal, Charakterisierungen von Saturationsklassen fïr singulare Integrale vom Laplaceschen Faltungstyp, TH Aachen, 1966.

9. K. Yosida, Functional analysis, Grundlehren d. math. Wiss. vol. 123, Springer, Berlin, 1965.

Technological. University of Aachen, Germany 